\title{
Uso de los programas de apoyo a la exportación por parte de los negocios verdes de Bogotá-Región
}

\author{
Claudia Paola García-Castiblanco ${ }^{1}$ \\ Universitaria Agustiniana \\ claudia.garcia@uniagustiniana.edu.co \\ Diana Marcela Díaz-Ariza² \\ Universitaria Agustiniana \\ diana.diaza@uniagustiniana.edu.co \\ Estibaliz Aguilar-Galeano ${ }^{3}$ \\ Universitaria Agustiniana \\ estibaliz.aguilar@uniagustiniana.edu.co
}

\section{Fecha de recepción: 23 de junio de 2020 \\ Fecha de aprobación: 11 de marzo de 2021 \\ Fecha de publicación: 10 de mayo de 2021}

Cómo citar este artículo / To reference this article / Comment citer cet article / Para citar este artigo:

García-Castiblanco, C. P.; Díaz-Ariza, D. M.; Aguilar-Galeano, E. (2020). Uso de los programas de apoyo a la exportación por parte de los negocios verdes de Bogotá-Región. Revista Escuela de Administración de Negocios, (89), 197-214.

DOI: https://doi.org/10.21158/01208160.n89.2020.2872

\section{Resumen}

El deterioro ambiental causado por la intensa actividad económica desarrollada desde el siglo pasado ha llevado a que en las últimas décadas se cuestionen las formas tradicionales de producción, de modo que se ha abierto el espacio a nuevas oportunidades de negocios en las que se realice un manejo responsable de los recursos. Esta nueva tendencia, que se consolida, principalmente, en Europa y Norteamérica, está marcada por la aparición de consumidores conscientes que se preocupan por que los procesos productivos sean amigables con el medio ambiente. En este contexto, los negocios verdes se proponen como una nueva alternativa de desarrollo para Colombia, sin embargo, poco se ha indagado sobre su potencial exportador. De acuerdo con la teoría de la competitividad ambiental, este estudio tiene como objetivo establecer qué tanto emplean los negocios verdes de Bogotá-Región programas gubernamentales de fomento a la exportación para llevar a cabo sus procesos de internacionalización. Este es un estudio de corte transversal de tipo correlacional que, por medio de encuestas, indaga la relación entre las variables planteadas en las compañías que han sido clasificadas como negocios verdes en Bogotá-Región por la Secretaría Distrital de Ambiente y

1 Profesional en Relaciones Internacionales - Colegio Mayor de Nuestra Señora del Rosario. Especialista en Política Internacional y Magíster en Asuntos Internacionales - Universidad Externado de Colombia. ORCID:

2 Economista - Universidad Nacional de Colombia. Magíster en Investigación en Problemas Sociales Contemporáneos - Universidad Central. ORCID: https://orcid.org/0000-0002-8606-3234

3 Bióloga - Universidad Nacional de Colombia. Especialista en Ambiente y Desarrollo Local - Universidad Francisco José de Caldas y Magíster en Desarrollo Humano de la Facultad Latinoamericana de Ciencias Sociales (FLACSO). ORCID: https://orcid. org/0000-0002-8942-1164 
la Corporación Autónoma Regional de Cundinamarca (CAR). Entre los hallazgos se encuentra que existe un bajo nivel de exportación y se evidencia poco conocimiento sobre los programas de apoyo existentes, lo cual afecta su uso.

Palabras clave: negocios verdes; procesos de internacionalización; nivel de exportación; potencial exportador; fomento a la exportación; competitividad ambiental; procesos productivos ecoamigables.

\title{
How green businesses in Bogota-Region use export support programs
}

\begin{abstract}
The environmental deterioration caused by the intense economic activity that has been taking place since last century has led to the questioning of traditional forms of production in recent decades, opening the way to new business opportunities in which resources are responsibly managed. This new trend, which is consolidating its position mainly in Europe and North America, is marked by the emergence of conscious consumers who are concerned about environmentally friendly production processes. In this context, green businesses are proposed as a new alternative for development in Colombia; however, little research has been done on their export potential. According to the theory of environmental competitiveness, this study aims at establishing the extent to which green businesses in Bogota-Region use government export promotion programs to carry out their internationalization processes. This is a cross-sectional correlational study that, by means of surveys, investigates the relationship between the variables raised in the companies that have been classified as green businesses in Bogota-Region by the District Secretariat of Environment and the Regional Autonomous Corporation of Cundinamarca (CAR, by its acronym in Spanish). Among the findings, there is a low level of exportation and there is little knowledge about the existing support programs, situation that affects their use.
\end{abstract}

Keywords: green business; internationalization processes; export level; export potential; export promotion; environmental competitiveness; eco-friendly production processes.

\section{Uso de programas de apoio à exportação por empresas verdes na Região de Bogotá}

\section{Resumo}

A deterioração ambiental ocasionada pela intensa atividade econômica desenvolvida desde o século passado tem levado a questionar, nas últimas décadas, as formas tradicionais de produção, de modo que tem se aberto o espaço para novas oportunidades de negócios em que é realizada a gestão responsável dos recursos. Essa nova tendência, que se consolida principalmente na Europa e na América do Norte, é marcada pelo surgimento de consumidores conscientes que se preocupam com os processos de produção para que sejam amigáveis ao meio ambiente. Nesse contexto, os negócios verdes são propostos como uma nova alternativa de desenvolvimento para a Colômbia, porém, pouco se pesquisou sobre seu potencial exportador. De acordo com a teoria da competitividade ambiental, este estudo tem como objetivo estabelecer o quanto as empresas verdes da Região de Bogotá utilizam programas governamentais de promoção de exportações para realizar 
seus processos de internacionalização. Trata-se de um estudo transversal de natureza correlacional que, por meio de questionários, estuda a relação entre as variáveis levantadas nas empresas que foram classificadas como negócios verdes na Região de Bogotá pela Secretaria Distrital de Meio Ambiente e pela Corporação Autônoma Regional de Cundinamarca (CAR). Entre as constatações está que existe um baixo nível de exportação e pouco conhecimento é evidenciado sobre os programas de apoio existentes, o que afeta a sua utilização.

Palavras-chave: negócios verdes; processos de internacionalização; nível de exportação; potencial de exportação; promoção de exportação; competitividade ambiental; processos de produção ecológicos.

\section{De l'utilisation des programmes d'aide à l'exportation pour les entreprises écoresponsables de Bogotá et sa région}

\section{Résumé}

La dégradation de l'environnement et des écosystèmes, provoquée par les activités économiques et humaines depuis plusieurs décennies, a conduit à remettre en cause les modèles de production des dernières décennies, ouvrant de nouvelles opportunités commerciales aux entreprises exerçant une gestion responsable des ressources. Cette nouvelle tendance, qui se consolide principalement en Europe et en Amérique du Nord, est marquée par l'apparition de consommateurs conscients et soucieux du respect de l'environnement lors des processus de production. C'est dans ce contexte que de nouvelles alternatives de création et développement d'entreprises vertes apparaissent en Colombie même si les études de leur potentiel à l'exportation sont encore peu nombreuses. Conformément à la théorie de la compétitivité environnementale, cette étude vise à déterminer dans quelle mesure les entreprises écoresponsables de la région de Bogotá utilisent les programmes gouvernementaux de promotion des exportations pour mener à bien leurs processus d'internationalisation. Il s'agit d'une étude transversale de type corrélationnel qui analyse les relations entre les variables des entreprises écoresponsables de la région de Bogotá, le secrétariat à l'environnement et la société autonome régionale de Cundinamarca (CAR). Les résultats montrent un faible niveau d'exportation et un manque de connaissances et d'utilisation des programmes d'aide existants.

Mots-clés: entreprises vertes; processus d'internationalisation; niveau d'exportation; potentiel d'exportation; promotion des exportations; compétitivité environnementale; processus de production respectueux de l'environnement. 


\section{Introducción}

T os procesos de internacionalización dependen, Uen gran medida, de las características de las empresas: su antigüedad, su tamaño y el sector al que pertenecen, entre otros (Álvarez, 2004; Ayob y Freixaned, 2014; Langseth, O'Dwyer y Arpa, 2016; Nguyen, Le y Bryant, 2013). Colombia es un país que viene incrementando de manera constante el número de empresas exportadoras, de modo que había alcanzado 11292 para diciembre del 2017, pero los productos que más se venden en el extranjero son aún de explotación minera o agrícola (Analdex, 2018), lo que muestra que existen actividades que, a pesar de su potencial, mantienen una participación marginal en las exportaciones del país.

Los negocios verdes, por ejemplo, ofrecen nuevas oportunidades para emprendedores innovadores que pueden aprovechar el espacio que se le ha generado a los productos hechos a partir del uso sostenible de la biodiversidad o con técnicas más amigables con el ambiente, como respuesta al grave deterioro ambiental que se ha producido en las últimas décadas, en razón a las técnicas de producción y las tendencias de consumo tradicionales.

Estudios como los de Porter y van der Linde (1995) proponen que este tipo de productos pueden adquirir lo que se ha denominado competitividad ambiental, la cual se genera cuando las empresas se ven obligadas a innovar en la forma que desarrollan sus procesos, a fin de cumplir con las regulaciones ambientales, de manera que obtienen, además, la oportunidad de abrir nuevos mercados en aquellos países en los que la legislación en la materia es más flexible.

Sin embargo, en la actualidad se encuentra que existen empresas que producen de manera amigable con el medio ambiente, de forma voluntaria y con estándares superiores a los establecidos por la ley (González-Benito y González-Benito, 2006), las cuales buscan aprovechar las preocupaciones por las cuestiones ambientales y los cambios en las tendencias de consumo (Chen y Chang 2012; Leonidou et al. 2015).
En este contexto, la Organización de las Naciones Unidas - ONU - ha sido un importante impulsor de esta tendencia a través de la realización de estudios sobre la materia, como, por ejemplo, el informe Brundtland de 1987 —también conocido como Nuestro futuro común-, el desarrollo de la Cumbre de la Tierra en 1992, la generación de la iniciativa BioTrade en 1996 con el objetivo de promover el comercio de bienes y servicios que deriven de la explotación sostenible de recursos naturales, y, de manera más reciente, el establecimiento de los Objetivos de Desarrollo Sostenible (ODS) en el 2016, entre los cuales el número 12 se enfoca de manera específica en la producción y el desarrollo sostenible.

La gestión eficiente de los recursos naturales y la forma en que se eliminan los desechos tóxicos y los contaminantes son vitales para lograr este objetivo. También es importante instar a las industrias, los negocios y los consumidores a reciclar y reducir los desechos, como asimismo apoyar a los países en desarrollo a avanzar hacia patrones sostenibles de consumo para el 2030. (Programa de las Naciones Unidas para el Desarrollo [PNUD], 2016)

En Colombia, los esfuerzos en esta materia empiezan a hacerse visibles con el establecimiento, en el 2012, del Plan Nacional de Negocios Verdes (PNNV), los programas regionales de negocios verdes y el Documento CONPES 3934 —Consejo Nacional de Política Económica y Social_-sobre crecimiento verde. Iniciativas de carácter público que buscan consolidar la oferta y la demanda de estos productos tanto en mercados nacionales como internacionales (Ministerio de Ambiente y Desarrollo Sostenible, $2014 b)$. Además de estos programas, desde el inicio de la apertura económica en la década de los noventa se crearon los entes encargados de la promoción de los productos nacionales en el extranjero. Para desarrollar dicha labor existen dos entidades que tienen una relación más directa con los exportadores en la búsqueda por consolidarse en su actividad internacional: el Grupo Bancoldex y Procolombia. 
Como se aprecia, existe toda una estructura de apoyos que pueden ser empleados por quienes quieran desarrollar iniciativas de negocios ambientalmente sostenibles y deseen internacionalizar sus productos. Es claro que, para los entes gubernamentales, estimular el emprendimiento se convierte en una alternativa de desarrollo que genera empleo y riqueza (Marulanda-Valencia y Morales-Gualdrón, 2016).

Por tanto, teniendo en cuenta que una vez hecha la revisión de la literatura se encontró que son pocos los estudios que analizan la relación entre los programas de apoyo a las exportaciones con las exportaciones de productos amigables con el medio ambiente (Kanda, Mejía-Dugand y Hjelm, 2015; Kanda, Hjelm y MejíaDugand, 2016), y que no se registran documentos que abordan el tema en Colombia, esta investigación tiene por objetivo establecer qué tanto emplean los negocios verdes de Bogotá-Región los programas gubernamentales de fomento a la exportación para llevar a cabo sus procesos de internacionalización.

En este propósito, el presente artículo busca caracterizar los negocios verdes de Bogotá-Región para identificar los que han desarrollado o desarrollan exportaciones, definir el nivel de conocimiento y el uso de los programas de apoyo a las exportaciones, así como evidenciar si dichos apoyos inciden en la internacionalización de los negocios verdes.

Con la finalidad de desarrollar los objetivos propuestos se realizó un estudio de corte transversal y tipo correlacional en el que se aplicó un instrumento de recolección de información tipo encuesta a sesenta y cinco empresas calificadas como negocios verdes por la CAR Cundinamarca y la Secretaría Distrital de Ambiente, para lo cual se adaptó un modelo conceptual con el fin de tratar variables en el orden jerárquico diseñado por Ayob y Freixaned (2014).

Para su desarrollo, el presente documento se divide en siete secciones. La presente introducción que continua con la revisión de la literatura. En la segunda sección se realiza una breve explicación de lo que es la competitividad ambiental, en la tercera se aborda el tema de los negocios verdes y los programas de apoyo existentes en Colombia. La cuarta parte presenta la metodología, seguida de los resultados y la discusión, para cerrar en la séptima sección con las conclusiones.

\subsection{Revisión de la literatura}

Los programas de fomento a las exportaciones son una herramienta que permite a los empresarios conocer oportunidades de mercado y financiación para la comercialización de sus productos en el extranjero. Aunque en varios países - como, por ejemplo, en Colombia- existen programas orientados específicamente a la promoción de productos de negocios verdes, son escasos los estudios que han intentado verificar el impacto o la efectividad de este tipo de apoyos.

Se encuentra, por ejemplo, la clasificación de distintos tipos de programas que se han identificado en países diferentes tales como Suecia, Noruega o Estados Unidos (Kanda et al., 2016) y la efectividad en su aplicación. Este último aplica solo para el caso de Suecia, en donde la valoración concluyó que las empresas que producen tecnologías limpias poco conocen los instrumentos de apoyo, ya que el $62 \%$ responde no ser consciente de su existencia, lo cual puede ser un problema de divulgación efectiva; ahora, quienes los conocen pero no los emplean mencionan las diferentes trabas burocráticas para acceder a ellos, mientras que quienes han participado lo han hecho porque encontraron alguna iniciativa acorde a sus necesidades. De igual modo, el estudio apunta que quienes aún no exportan es porque a) tienen un buen nicho dentro del país, b) no les interesa, o c) no tienen la capacidad para hacerlo. En cuanto al conocimiento de los instrumentos de apoyo se encuentra que es bajo (Kanda et al., 2015).

La dificultad de encontrar estudios dirigidos específicamente al apoyo de negocios verdes se debe, en gran medida, a que, por lo general, estos programas de fomento a las exportaciones suelen tener un campo de análisis más amplio en el sentido que no tienden a limitarse a un tipo de producto, sino a estudios según el tamaño de la empresa, a nivel nacional o regional, indagando sobre su forma de funcionamiento e incluso a medir su efectividad en diferentes países como, por ejemplo, Chile (Álvarez, 2004; Volpe y 
Carballo 2010), Argentina (Volpe, Carballo y García, 2012), Brasil, (Ferreira-Dornela, y Teixeira-Carneiro, 2018), Colombia (Sierra, 2009), España (Cansino et al., 2013; Freixanet, 2012) y Malasia (Ayob y Freixaned, 2014), por mencionar algunos.

Respecto a su forma de funcionamiento, aunque pueden tener diversas clasificaciones (Álvarez, 2004; Ferreira-Dornela y Teixeira-Carneiro, 2018), es posible encontrar elementos comunes en los tipos de instrumentos o en las ayudas ofrecidas por los gobiernos que se pueden resumir en asesorías, acceso a información, participación en ferias o ruedas de negocios y apoyos financieros, aunque las denominaciones que se empleen para su clasificación difieran en cada país e investigación.

En cuanto a su efectividad, los resultados son variados y se encuentran apreciaciones como, por ejemplo, que las empresas pequeñas y con poca experiencia se benefician más de los programas de apoyo (Broocks y Van Biesebroeck, 2017; Calle y Tamayo, 2005; Cansino et al., 2013; Volpe y Carballo, 2010, Wilkinson y Brouthers, 2006) o que las pymes buscan más los apoyos en asesorías que los financieros (Ayob y Freixaned, 2014; Cansino et al., 2010). Las pymes con apoyo obtienen un mayor logro de sus objetivos financieros (Durmuşoğlu et al., 2012) o crecimiento en sus exportaciones (Volpe y Carballo, 2010), y los programas, además, permiten tener un mejor conocimiento de los procesos para exportar al disminuir las dificultades que se presentan en las empresas que inician sus actividades exportadoras (Cansino et al., 2013).

También se ha buscado identificar que tanto se conocen los programas de apoyo y su relación con las empresas exportadoras y no exportadoras (Kanda et al., 2015; Ayob y Freixaned, 2014), de manera que las empresas exportadoras tienen más conciencia del conocimiento y beneficio que puede generar el empleo de estos programas o la adquisición de nuevas habilidades como, por ejemplo, las administrativas, el acceso a recursos informativos, la identificación de mercados clientes y de tipo financiero (Álvarez, 2004; Durmuşoğlu et al., 2017).
En el caso colombiano, la mayoría de las empresas han iniciado su proceso de internacionalización a partir de la década de los noventa a través de exportaciones, como resultado de la toma de decisiones gerenciales que la identifican como una estrategia ofensiva hacia la competencia (Vélez-Ocampo y González Pérez, 2015).

Además, como lo indican las teorías de la internacionalización, las compañías más maduras son las que se lanzan a desarrollar estos procesos, aunque requieren aumentar sus esfuerzos en innovación, investigación y renovación tecnológica, entre otros, para mejorar su competitividad (Calle y Tamayo, 2005). Sin embargo, se encuentra que, para las pymes colombianas, la exportación no es percibida como un indicador de desarrollo o crecimiento, pero las empresas medianas tienen mejor desempeño que las pequeñas (Sierra, 2009).

Lo anterior puede explicarse a partir de la teoría de la rigidez lateral, esbozada por Luostarinen (1979) y retomada por Tan, Brewer y Liesch (2018), la cual explica cómo, a pesar de lo propuesto por teorías como Uppsala, que abordan la internacionalización como un proceso progresivo y natural, se encuentran algunas empresas que, pese a su madurez, no responden a los estímulos, de manera que son más relevantes las dificultades o los temores percibidos tales como los costos o la falta de conocimientos que los probables beneficios. En otras palabras, las empresas, en vez de ir hacia adelante - lo que sería la exportación-, mantienen movimientos laterales.

La investigación de Tan et al. (2018) propone que existen cuatro factores por los que las empresas se resistirían a iniciar sus procesos exportadores - o de internacionalización-: a) orientación doméstica, lo que significa que la prioridad está en el mercado doméstico; b) limitación de estímulos, según la cual, no hay mucho interés por parte de los tomadores de decisiones por conocer las políticas de estímulos a la exportación; c) conocimientos y experiencia limitadas en lo concerniente a procesos exportadores; y d) inercia, según la cual hay «falta de respuesta de 
un decisor ante los estímulos debido a que está satisfecho con el estado actual de la empresa» (p. 7). Estos factores pueden ayudar a explicar el por qué negocios con potencial exportador se mantienen en sus mercados nacionales.

\section{Competitividad ambiental}

$\mathrm{P}$ orter y Van der Linde (1995) proponen la competitividad ambiental como la posibilidad de que las empresas que desarrollen procesos amigables con el medio ambiente puedan verse beneficiadas en términos de acceso a mercados internacionales. $\mathrm{Si}$ bien en el inicio pueden verse obligadas a innovar en la forma que desarrollan sus procesos para cumplir con las regulaciones ambientales, esto puede redundar en la capacidad de abrir nuevos mercados en países donde la legislación en la materia es más flexible.

De esta forma, la adopción de prácticas sostenibles por parte de las empresas genera beneficios de diversos tipos que pueden tener incidencia en el fortalecimiento de su competitividad a través, por ejemplo, del ahorro, resultado de un uso más óptimo de los recursos, así como pueden mejorar su potencial innovador al cambiar el desarrollo de sus procesos o al generar nuevos productos (Porter y Van der Linde, 1995). La teoría de la competitividad ambiental parte del principio de que las empresas modifican su conducta ambiental como una forma de dar respuesta a las regulaciones establecidas para tal fin. Sin embargo, esta visión corresponde a lo que hoy en día se denomina «una empresa reactiva».

De esta forma, sí las empresas solo se adaptan a la regulación ambiental del país del que provienen, se trata de un negocio de tipo reactivo (GonzálezBenito y González-Benito, 2016), pero si la adopción de prácticas ambientales es voluntaria y va más allá de lo exigido por la ley, son negocios proactivos (Lannelongue, González-Benito y González-Benito, 2014; González-Benito y González-Benito, 2016), ya que responden a las preocupaciones sociales por el cuidado del medio ambiente y lo asumen como una oportunidad para potencializar su negocio.
Se requiere, entonces, características como la innovación o la creatividad verde para estar en capacidad de desarrollar productos en esta línea (Chen, y Chang, 2013; Garcez, Hourneaux-Junior y Farah, 2016). Aunque en cualquiera de las dos condiciones es posible que las empresas desarrollen competitividad ambiental, estudios demuestran que la proactividad influye positivamente en la adquisición de este tipo de competitividad y, a su vez, en el desempeño exportador (Aragón-Correa, et al., 2008; Leonidou et al., 2015; Martín-Tapia, AragónCorrea y Senise-Barrio, 2008).

Los negocios verdes, de acuerdo con la clasificación del PNNV, tenderán a ser empresas proactivas. Los estudios que se han generado en la materia han identificado ciertas características que llevan a la proactividad, como, por ejemplo, las iniciativas y las ideologías de sus directivos (Borial et al., 2013; Kashmanian, Keenan y Wells, 2010; Spence, Boubaker y Ondoua, 2011), por adaptación a escenarios internacionales (Chen y Chang, 2012; Chen y Chang 2013) o por tener una buena imagen o reputación frente a los stakeholders, a fin de dar una respuesta rápida a las preocupaciones medioambientales, además de considerarlo un factor diferenciador (Martín-Tapia et al., 2008; Leonidou et al., 2015).

En esta línea también se encuentran estudios que muestran cómo invertir en tecnologías limpias genera una relación entre productividad y el incremento de exportaciones, ya que amplía la posibilidad de aumentar la creación de nuevos productos y penetrar a nuevos mercados con mayor regulación ambiental (Antonietti, y Marzucchi, 2014), tal como lo indica la teoría. 
Por tanto, una actitud ambiental y proactiva por parte de las empresas puede tener una incidencia positiva sobre el comportamiento exportador, ya que logran desarrollar ventajas intangibles, como, por ejemplo, la buena reputación en la calidad de los procesos de producción, la cual puede ser certificada por normas internacionales como la ISO 14000, de manera que se obtienen reconocimiento $y$ beneficios por esto (de Vries, Bayramoglu y van der Wiele , 2012). Sin embargo, las ayudas por parte del Estado, así como los programas de apoyo a los exportadores, pueden ser también un instrumento que le permita a los negocios verdes acceder a mercados en el extranjero, ya que la internacionalización requiere de conocimiento, acceso a la información e, incluso, asistencia financiera para su realización (Ayob y Freixaned, 2014).

\section{Negocios verdes en Colombia}

L as empresas son llamadas cada vez más a tomar un papel proactivo en el desarrollo sostenible, ya que, de acuerdo con el Pacto Mundial (United Nations Development Group, 2014), se ha identificado que la era posterior al 2015 representa una oportunidad histórica para que la comunidad empresarial internacional contribuya al logro de los objetivos mundiales de sostenibilidad y desarrollo.

Es por esto que el sector empresarial se presenta ahora como un gran actor del desarrollo, el cual, junto con donantes, gobiernos y diversas ONG, lidera las iniciativas dirigidas a mejorar la vida de las personas. Para esto, la función de la empresa como actor de desarrollo está relacionado con aspectos referidos a innovación, recursos, capacidad y liderazgo; el papel central, entonces, se encuentra en promover el avance de la innovación, promoviendo organizaciones que ayuden a la creación de ingresos y empleos, movilizando recursos internos y, a su vez, contribuyendo a la reducción de la pobreza y de las problemáticas ambientales (Scheyvens, Banks y Hughes, 2016).

En Colombia, el Gobierno nacional, en cabeza del Ministerio de Ambiente y Desarrollo Sostenible, formularon en el 2012 el PNNV con el fin de « definir los lineamientos y proporcionar herramientas para la planificación y la toma de decisiones que permitan el desarrollo, el fomento y la promoción tanto de la oferta como de la demanda [de este tipo de negocios en el país]». (Ministerio de Ambiente y Desarrollo
Sostenible, 2014a, p. 49) Los negocios verdes son entendidos, de acuerdo con el PNNV, como actividades económicas asociadas a la producción y la oferta de bienes o servicios que generan impactos ambientales positivos, contribuyendo a la conservación del ambiente como capital natural que soporta el desarrollo.

Como se específica en el PNNV, los negocios verdes en Colombia se clasifican en tres categorías y ocho sectores que son dinámicos y cuya reglamentación es dada por cada una de las autoridades competentes, dependiendo del tema, de modo que se dividen en categorías relacionadas con la producción de bienes y servicios provenientes del uso sostenible de los recursos naturales, o en negocios enfocados en la generación de bienes que generan impactos positivos en el medio ambiente, de manera que son menos contaminantes o ayudan a resolver impactos ambientales, y una última división relacionada con negocios que se enmarcan en los mercados de carbono.

La clasificación de una empresa como negocio verde, además de tener el enfoque de sostenibilidad y centrar su actividad económica en alguna de las tres divisiones mencionadas, debe cumplir con la mayoría de un conjunto de doce criterios que el mismo PNNV delimita, toda vez que el bien o servicio debe responder a un contexto de desarrollo sostenible en el que se procure no causar efectos indeseables en el ambiente o la sociedad, sin disminuir su calidad. 
Los criterios definidos en el PNNV evidencian estar organizados en el marco del enfoque de la triple línea, por el cual se plantea que el fortalecimiento de una empresa depende de tres áreas de actuación: una económica, otra social y una medioambiental (Rodríguez y Ríos-Osorio, 2016).

Desde esta mirada, se identifican los criterios de viabilidad económica —uno-, comunicación de atributos sociales o ambientales asociados al bien o servicio - once- y esquemas, programas o reconocimientos ambientales $\mathrm{o}$ sociales implementados o recibidos — doce-, los cuales se enfocan en aspectos económicos que buscan llevar a las empresas a tomar conciencia de factores que evidencien su desempeño e impacto financiero (Rodríguez y Ríos-Osorio, 2016).

Otro grupo de criterios aborda aspectos ambientales, los cuales se enfocan en los impactos generados directa e indirectamente por la empresa al llevar a cabo su proceso productivo (Rodríguez y RíosOsorio, 2016), en relación con lo cual se definen los criterios de impacto ambiental positivo del bien o servicio — dos-, enfoque de ciclo de vida del bien o servicio - tres-, vida útil — cuatro-, no uso de sustancias o materiales peligrosos - cinco-, reciclabilidad de los materiales o uso de materiales reciclados — seis—, y uso eficiente y sostenible de recursos para la producción del bien o servicio siete-, de forma que se haga la identificación y el control de los impactos generados y se logre un proceso productivo racional (Ministerio de Ambiente y Desarrollo Sostenible, 2014a).

En cuanto al aspecto social, los esfuerzos se enfocan en cómo las empresas ayudan a mejorar las condiciones tanto internas como externas de las sociedades con las que interactúan, en cualquier punto de la cadena de valor empresarial (Rodríguez y Ríos-Osorio, 2016). De esta forma, se definen los criterios responsabilidad social al interior de la empresa -ocho-, responsabilidad social y ambiental en la cadena de valor de la empresa - nueve- y responsabilidad social y ambiental al exterior de la empresa diez-, los cuales se enfocan en un trato ético y las condiciones de trabajo justas para los empleados, directos y accionistas, así como en las buenas prácticas con proveedores, servicios tercerizados y clientes, en relación con la gestión de sus impactos sobre ámbitos de influencia externa, comunidades, Estado, consumidores y la sociedad en general (Ministerio de Ambiente y Desarrollo Sostenible, 2014a).

Con el fin de promocionar los negocios verdes en Colombia se han establecido estrategias tales como los programas regionales de negocios verdes, por los cuales se busca «brindar los parámetros para orientar, articular y contribuir a la implementación, consolidación y fortalecimiento de la oferta y demanda de productos y servicios verdes a nivel regional, nacional e internacional» (Ministerio de Ambiente y Desarrollo Sostenible, 2014b). Estas estas estrategias propenden a identificar y priorizar productos verdes regionales con calidad de exportación que aprovechen las oportunidades brindadas por los TLC, así como a fortalecer la oferta exportadora de ciertos productos verdes priorizados, relacionados, principalmente, con biocomercio y agrosistemas sostenibles.

\subsection{Programas de apoyo a la exportación en Colombia}

En Colombia, las entidades encargadas de apoyar a los exportadores son el Grupo Bancoldex y ProColombia. El Grupo Bancoldex está conformado por tres instituciones: Bancoldex, Fiducoldex y Arco. Bancoldex es una entidad financiera creada en 1991. Entre sus propósitos se encuentran promover el crecimiento del comercio exterior e incrementar la competitividad y la productividad enfocándose de manera particular en las mipymes. La entidad maneja diferentes líneas de crédito, y es la línea de apoyo al sector exportador/importador la directamente relacionada con el apoyo a los exportadores. Bancoldex también brinda ayudas no financieras que incluyen la consultoría y la formación alrededor de temas tales como la cobertura cambiaria, los diagnósticos para mejorar la competitividad y la gestión financiera, entre otros. 
Fiducoldex, por su parte, ofrece líneas de crédito a los exportadores, lo que corresponde a un esquema fiduciario de fuente de pago mediante el cual los pagos que reciben los empresarios por las ventas en el exterior son empleados para pagar la obligación crediticia. Argo, por último, realiza operaciones de leasing con el objetivo de captar dinero y ofrecer préstamos, aunque no se identifica una línea específica para exportadores.

Ahora bien, ProColombia -antes Proexporttambién creada en 1991 y puesta en marcha en 1992, tiene como propósito la promoción de las exportaciones, la inversión extranjera y el turismo a través de acciones tales como la identificación de opciones de mercado, el diseño de estrategias de penetración de mercados, la internacionalización de las empresas y el acompañamiento en el diseño de planes de acción, entre otros. Para la obtención de estos objetivos, Procolombia desarrolla diversas actividades, como, por ejemplo, asesorías, capacitación, macrorruedas, ruedas de negocios, ferias internacionales, showrooms y elaboración de planes de trabajo conjunto.

En cuanto a la promoción de la exportación de productos verdes, en un principio el Ministerio de Ambiente y Desarrollo Sostenible abordó la temática a través de dos programas: el PNNV, lanzado en el 2012, y el Programa Nacional de Biocomercio Sostenible en el 2014. Ambos cuentan con propuestas de estímulos y ayudas financieras para promover este tipo de actividad. Sin embargo, de manera reciente, ha sido el Departamento Nacional de Planeación (DNP) la entidad que ha establecido las líneas de trabajo para llevar al país a un sistema productivo sostenible y competitivo con miras al 2030. Para tal fin, en el 2018 creó la Misión de Crecimiento Verde, la cual reunió a un grupo de expertos con el objetivo de realizar un diagnóstico sobre el estado del biocomercio en el país, el cual da cuenta del potencial que existe en el área y lo poco desarrollada que está.

Además, el DNP instituyó el documento CONPES 3934, el cual define la Política de crecimiento verde -2018-2030 - para conducir al país a un modelo económico sostenible y alineado con los objetivos de desarrollo sostenible. En este sentido define cinco ejes de acción: a) diversificar la economía a partir de la producción de bienes y servicios producto del uso sostenible de los recursos naturales; b) reducir los impactos ambientales y sociales de las actividades productivas, haciendo un uso eficiente de los recursos; c) generación y fortalecimiento del capital humano; d) desarrollo de acciones estratégicas en materia de ciencia, tecnología e innovación; y e) asegurar la coordinación y articulación institucional (Departamento Nacional de Planeación, 2018).

Con base en lo anterior se propone la siguiente hipótesis:

- H1: la internacionalización de la empresa tiene relación con los apoyos recibidos.

\section{Metodología}

Para lograr el objetivo propuesto se desarrolló un estudio de corte transversal, de tipo correlacional, en la medida que se buscó indagar por la relación entre variables, en este caso la recepción de apoyos gubernamentales y el grado de internacionalización de las organizaciones.
La investigación se realizó con empresas verdes de Bogotá-Región, para lo cual se ubicó a las entidades gubernamentales encargadas de realizar los procesos de verificación de este tipo de empresas. De esta manera, la Corporación Autónoma Regional de Cundinamarca y la Secretaría Distrital de 
Ambiente de Bogotá proveyeron las bases de datos correspondientes a empresas verdes de la zona señalada; la Secretaría de Ambiente de Bogotá cuenta con veintiséis organizaciones registradas como negocios verdes, mientras que la CAR Cundinamarca tiene 281 empresas en dicha base.

Sin embargo, al realizar la verificación de la existencia de las empresas, vía telefónica, vía correo electrónico e indagando sobre su existencia a través de internet, se obtuvo que son 190 empresas de la CAR Cundinamarca las que están en funcionamiento, lo que arroja un total de 216 negocios verdes para Bogotá-Región.

Una vez obtenidos los datos de contacto de las empresas verdes para Bogotá-Región, se elaboró un instrumento de recolección de información tipo encuesta cuya estructura se dio de la siguiente manera: datos generales de la empresa que incluyen actividad económica, tiempo de funcionamiento y número de empleados; aspectos relacionados con la gerencia - género, formación, edad, experiencia en el cargo-; aspectos de internacionalización —etapa en la que se encuentra, razones para exportar o no hacerlo, destinos de exportación, conocimiento de programas para el fomento de exportación-, y sobre recepción de apoyos — si recibe apoyo o no, tipos de apoyo, cantidad de veces que se ha recibido, de dónde proviene el apoyo-.

En particular, para el diseño de las preguntas referidas a los aspectos de internacionalización y la recepción de apoyos, se tomó como base el estudio desarrollado por Ayob y Freixaned (2014), con el fin de estar en capacidad de analizar la información bajo la metodología de variables de orden jerárquico que se adaptó de su estudio.
El instrumento se aplicó en una primera etapa por correo electrónico. Debido a que la tasa de respuesta no fue tan alta se decidió empezar a aplicar la encuesta vía telefónica, a través de mensajería instantánea Whatsapp y Messenger de Facebook-, y en algunos casos se realizó de manera presencial —en los casos en los que solo se tenía el dato de la dirección de la empresa-. En total se logró recabar información completa por parte de sesenta y cinco empresas, lo cual da una tasa de respuesta del $30 \%$. Se definió, en primer lugar, aplicar una prueba no paramétrica chi cuadrado Pearson para identificar la relación de independencia de las variables internacionalización y apoyos recibidos.

Además, a fin de establecer qué tanto emplean los negocios verdes de Bogotá-Región los programas gubernamentales de fomento a la exportación para llevar a cabo sus procesos de internacionalización, como se mencionó, se realizó una adaptación de la metodología propuesta por Ayob y Freixaned (2014) en el estudio «Insights into public export promotion programs in an emerging economy: the case of Malaysian SMEs», quienes proponen un modelo conceptual para tratar las variables en orden jerárquico, a partir del cual las preguntas relacionadas a la información que se requiere —en este caso sobre exportaciones y conocimiento de programas de fomento de exportación - se realizan en orden secuencial y el tipo de respuesta se establece de forma dicotómica. En este orden de ideas, la metodología permite profundizar de manera progresiva en la información, así como describir en orden secuencial lo encontrado. 


\section{Resultados}

T as empresas con las que se desarrolló el Jestudio son en su totalidad mipymes, $82 \%$ microempresas y $18 \%$ pequeñas empresas, en su mayoría con una importante trayectoria en el mercado, pues el $40 \%$ corresponde a empresas con entre cinco y diez años de funcionamiento, el $20 \%$ a más de diez años funcionando y el $40 \%$ entre uno y cinco años en el mercado. Un $23 \%$ manifestó que su actividad económica es del sector primario, y en igual porcentaje al terciario, el $6 \%$ al secundario; un $48 \%$ no sabe o no respondió el sector de la actividad económica.

Respecto a características de los gerentes, el $58 \%$ son dirigidas por hombres y el $42 \%$ por mujeres. En su mayoría, los gerentes son personas adultas jóvenes: el $31 \%$ se ubica en el rango de los treintaiuno a los cuarenta años, el $26 \%$ entre cuarentaiuno y cincuenta años, otro $26 \%$ son mayores de cincuenta años y un $17 \%$ jóvenes entre veinte y treinta años. Acerca de su formación, la mayoría son profesionales - $62 \%$-, seguidos de los técnicos o tecnólogos -23\%-, algunos con educación media -6\%-, educación primaria $-6 \%-\mathrm{y}$ no registraron su respuesta $-3 \%$ -

Sobre los procesos de internacionalización se buscó indagar varios asuntos. En primer lugar, si están exportando o no, si han intentado exportar, su conocimiento sobre programas de exportación y el uso de estos. $\mathrm{Al}$ respecto se encontró que el $91 \%$ de estas empresas no ha exportado, el $6 \%$ exporta actualmente y un $3 \%$ exportó en algún momento, pero no ha vuelto a hacerlo.

En las empresas que no se ha realizado exportación se indagó por las razones que los llevan a esta situación. La tabla 1 resume lo encontrado con los empresarios.

Tabla 1. Razones de no exportar

\begin{tabular}{|l|c|}
\hline \multicolumn{1}{|c|}{ Razón } & Porcentaje \\
\hline Su orientación es hacia el mercado doméstico & $39 \%$ \\
\hline $\begin{array}{l}\text { Hay conocimiento y experticia limitada o temor sobre procesos } \\
\text { de exportación }\end{array}$ & $24 \%$ \\
\hline $\begin{array}{l}\text { Considera que sus condiciones técnicas o producción no permiten } \\
\text { iniciar el proceso }\end{array}$ & $19 \%$ \\
\hline La empresa está satisfecha con su situación actual & $10 \%$ \\
\hline No hay interés de usar programas de apoyo a la exportación & $7 \%$ \\
\hline No sabe/no responde & $2 \%$ \\
\hline
\end{tabular}

Fuente. Elaboración propia.

En general, se encuentra que para la mayoría de quienes no exportan la razón está asociada a la orientación doméstica que pueda tener la empresa, por lo cual, naturalmente, el horizonte de exportación no hace parte de su proyección estratégica. Sin embargo, cabe considerar el porcentaje relevante de empresas para las que las razones tienen que ver con limitaciones de conocimiento o de producción que permitan asumir el reto; es en este punto que los programas de apoyo gubernamental para exportación tendrían opción de aportar a la consolidación de estas posibilidades en dichos negocios. A pesar de la situación de no estar exportando y no tener la intención de hacerlo, al menos en el corto o mediano plazo, el $37 \%$ de los empresarios considera que exportar es una actividad importante con miras al desarrollo del negocio. 
Aunque son pocos los negocios que exportan, se indagó por los destinos de sus ventas en el comercio internacional, encontrando que la mayoría exportan a otros países del continente - $75 \%$ - y Europa $-25 \%$. Sobre los que en algún momento exportaron, pero actualmente no lo hacen, las razones de haber parado este proceso tienen que ver, fundamentalmente, con que no obtuvieron las ganancias que esperaban - $50 \%$ -

Sobre el conocimiento de programas de apoyo a exportación por parte del gobierno, la mayoría de los empresarios manifestaron no conocerlos -72 $\%$-, y del $28 \%$ que manifestó conocer al menos un programa de apoyo, el $83 \%$ no ha hecho uso de tales programas. El programa con mayor reconocimiento por parte de estos empresarios es ProColombia.

Respecto a apoyos recibidos por parte del gobierno, el $54 \%$ manifiesta no haber sido beneficiario de ningún apoyo, contrastando con un $46 \%$ que respondió afirmativamente en este ítem. Entre quienes respondieron haber recibido apoyo, la mayoría — $87 \%$ — indicó que el tipo de apoyo recibido fue no financiero, entre estos se tienen capacitaciones, acompañamientos en el ordenamiento del negocio y asesoría para la obtención de certificaciones, formación a gerentes, desarrollo de vínculos en redes o agremiaciones, entre otros. El $13 \%$ de quienes recibieron apoyos manifestó haber recibido apoyo financiero, particularmente en forma de créditos blandos o incentivos tributarios.

Como resultado del apoyo, los empresarios indicaron qué cambios habían percibido en su negocio luego de su recepción. Se les orientó para que eligieran tantos resultados como consideraran pertinentes entre incremento de ventas, reducción de costos, apertura de mercados, generación de nuevos productos, mejoras en procesos de producción, posibilidad de exportar, formalización de la empresa o no percibir cambios. En la tabla 2 se sintetiza la información suministrada por los empresarios respecto a este ítem. Los empresarios dan cuenta de la importancia de los apoyos al indicar en un porcentaje considerable -37 $\%$ - más de dos cambios percibidos.

Tabla 2. Resultados del apoyo

\begin{tabular}{|c|c|}
\hline Número de resultados indicados & Porcentaje \\
\hline Uno & $30 \%$ \\
\hline Dos & $30 \%$ \\
\hline Tres & $17 \%$ \\
\hline Cuatro o más & $20 \%$ \\
\hline No percibe cambios & $3 \%$ \\
\hline
\end{tabular}

Fuente. Elaboración propia.

A fin de probar la hipótesis propuesta sobre la relación entre las variables exportación y recepción de apoyos, se realizó una prueba no paramétrica chi cuadrado Pearson, dada la no normalidad de las variables. Se realiza el contraste de hipótesis, de manera que estas son:

- Ho: exportar es independiente de recibir apoyo;

- H1: exportar es dependiente de recibir apoyo.
En este caso, el resultado indica un P-valor de 0,03934, el cual es menor al 0,05 del valor de referencia, por lo que no se puede rechazar la hipótesis nula, es decir, no se rechaza la hipótesis de que el hecho de exportar sea independiente del hecho de recibir apoyos gubernamentales para el desarrollo del negocio.

Por último, en la tabla 3 se encuentran los pesos de cada una de las modalidades de cada variable. Estos pesos constituyen el indicador de la primera dimensión. 
Pares de modalidades de variables distintas con pesos positivos tendrán asociación positiva, en el sentido de que ocurren con mayor frecuencia, y pares de modalidades de variables distintas con pesos negativos estarán asociadas negativamente, en el sentido de que ocurren con menor frecuencia. En la columna contribución se encuentra el nivel de importancia, en términos porcentuales, de cada una de las modalidades.

Tabla 3. Pesos de cada una de las modalidades para las variables

\begin{tabular}{|c|c|c|c|}
\hline Variable & Modalidad & Peso & Contribución (\%) \\
\hline \multirow{3}{*}{ Nivel de internacionalización } & Exporta & 4,87 & 25,10 \\
\cline { 2 - 4 } & Exportó & 5,42 & 15,54 \\
\cline { 2 - 4 } & No exporta & $-0,53$ & 4,26 \\
\hline \multirow{3}{*}{ Razones de no exportar } & NA - no aplica- & 5,05 & 40,54 \\
\cline { 2 - 4 } & No vocación & $-0,70$ & 5,10 \\
\cline { 2 - 4 } & Sin condiciones & $-0,16$ & 0,12 \\
\hline \multirow{2}{*}{ Conocimiento programas apoyo exportación } & No & $-0,34$ & 1,40 \\
\cline { 2 - 4 } & Sí & 0,86 & 3,52 \\
\hline Uso prog. exportar & No & 0,00 & 0,00 \\
\hline \multirow{2}{*}{ Importancia de la exportación para el negocio } & Importante & 0,58 & 2,09 \\
\cline { 2 - 4 } & Poca o nula & $-0,33$ & 1,20 \\
\hline \multirow{2}{*}{ Recep. apoyos } & No & $-0,23$ & 0,49 \\
\cline { 2 - 4 } & Sí & 0,27 & 0,58 \\
\hline
\end{tabular}

Fuente. Elaboración propia.

De esta manera, tenemos que el indicador arroja los valores positivos más altos para aquellas empresas con nivel de internacionalización exporta o exportó, esto asociado a aquellas con conocimiento sobre programas de apoyo a la exportación que consideran importante la exportación para el negocio y recibieron apoyo. En contraposición encontramos asociadas aquellas que no exportan, que no tienen esa vocación sin conocimientos de programas de apoyo, que consideran poco importante la exportación y que no recibieron apoyo.

\section{Discusión}

¿s hallazgos permiten establecer que los que, en su mayoría, tienen una trayectoria superior a cinco años -60\%-, por lo que es probable afirmar, de acuerdo con teorías como Uppsala, que se encuentran en un nivel de madurez apropiado para iniciar sus procesos de internacionalización por medio de exportaciones. Sin embargo, el alto porcentaje - $92 \%$ - de empresas no exportadoras indica que algunas de estas compañías pueden estar en una etapa de rigidez lateral, según lo planteado por Luostarinen (1979).
Por esta razón, al indagar sobre las razones por las que no se ha tomado la decisión de exportar, a la luz de los factores establecidos por Tan et al. (2018) se encuentra que la mayoría de las empresas han priorizado su orientación doméstica, lo que puede significar que se sienten cómodas con la situación actual y la exportación, por tanto, implicaría un riesgo que no se desea afrontar. Estos resultados están en consonancia con lo hallado por Kanda et al., (2015) quienes también señalan que la principal razón para no exportar es que se tiene un buen nicho en el mercado nacional. 
No sorprende mucho los resultados sobre el conocimiento de los programas de apoyo a las exportaciones, ya que los altos índices de desconocimiento $-72 \%$ - coinciden con estudios previos como el de Kanda et al. (2015) en los que el porcentaje alcanzó el $62 \%$, muy cercano al nacional. Kanda et al. (2015) especulan que esto puede deberse a que falta divulgación, pese a que este criterio no fue evaluado en esta investigación. Como era de esperarse, las empresas exportadoras son mucho más conscientes de los programas de apoyo y para ellas la internacionalización es una actividad muy importante, lo que se encuentra en sintonía con lo hallado por Ayob y Freixaned (2014).

En cuanto a las empresas que sí han hecho uso de los apoyos gubernamentales a la exportación, la mayoría indicó que no buscaron apoyos financieros, sino que se inclinaron por las capacitaciones, las asesorías o el acceso a la información, lo que también está en consonancia con los estudios de Ayob y Freixaned (2014) y Cansino et al. (2010), pues, como lo explican Cansino et al., (2013), cuando una empresa inicia su actividad exportadora requiere más de apoyos alrededor de la adquisición de conocimientos sobre cómo afrontar los procesos que las ayudas de tipo económico, por lo que resulta lógico que el programa más reconocido sea el de ProColombia.

Sobre los apoyos recibidos para el desarrollo de negocios verdes, propios del PNNV o del Programa Nacional de Biocomercio, cabe destacar que, si bien en estas propuestas gubernamentales se ha dispuesto como objetivo importante el ingreso de este tipo de negocios en los mercados internacionales y, por tanto, se han creado diversos instrumentos de apoyo para el logro de dicha meta, estos parecen no ser tan efectivos.

$\mathrm{Al}$ igual que ocurre con los instrumentos orientados a la promoción de las exportaciones, aquellos propios de los negocios verdes parece que no han sido suficientemente difundidos, pues la mayoría - $54 \%$ - manifestaron no haber recibido alguna vez este tipo de ayudas. Lo anterior lleva a plantear una dificultad de comunicación entre las propuestas gubernamentales y el público objetivo.
Sin embargo, no menos importante es el tipo de apoyo que se brinda. En general, los instrumentos dispuestos por los programas orientados a negocios verdes son de tipo no financiero, lo que incluye capacitaciones sobre desarrollo organizacional, asesorías para formalizar el negocio, así como participación en ferias y eventos de promoción del negocio de tipo local.

El resultado obtenido podría explicarse en la medida en que no se encontró, particularmente, mención a ningún apoyo que estuviera orientado al estudio o la promoción del negocio en mercados internacionales, lo cual se considera un aspecto relevante a la hora de apoyar la internacionalización de las empresas, ya que, como mencionan Ayob y Freixaned (2014) y Cansino et al. (2010), para estas pequeñas empresas se considera más relevante la ayuda técnica para conocer el entorno del mercado en el exterior que, incluso, la ayuda financiera dirigida a acceder a este.

Otra explicación que puede aducirse para el bajo desempeño exportador de estos negocios tendría que ver con el tipo de mercados hacia los cuales esté orientando el negocio. Aunque, conforme a los resultados, son pocas las empresas estudiadas que han tenido una experiencia exportadora, las que lo han hecho se han orientado, en su mayoría, al mercado cercano $-75 \%$ ha exportado a otros países del continente-. Siguiendo los referentes de competitividad ambiental de Porter y Van der Linde (1995), parte del éxito competitivo de la empresa radica en la eficiencia de la demanda, es decir, en la exigencia de la demanda local e internacional.

Respecto a esto, si existe un problema identificado en las barreras al crecimiento verde en esta zona del mundo es justamente la baja demanda que aún tienen este tipo de productos y servicios verdes, pues debido a los altos índices de desigualdad y pobreza, para el consumidor de América Latina aún priman elementos como el precio sobre los beneficios ambientales que estos puedan reportar (Cepal, 2017). Esto se asociaría, por ejemplo, al resultado encontrado en las empresas que alguna vez exportaron y no volvieron a hacerlo debido a que no obtuvieron las ganancias esperadas. 
Finalmente, en cuanto a la hipótesis propuesta y contrario a lo establecido en múltiples investigaciones (Álvarez, 2004; Broocks y Van Biesebroeck 2017; Volpe y Carballo, 2010, Wilkinson y Brouthers, 2006) en este trabajo no fue posible establecer una clara relación entre la recepción de apoyos y la internacionalización de la empresa. Esto abre el espacio para que en el futuro se indague el porqué.

\section{Conclusiones}

$\mathrm{A}$ unque la teoría de la competitividad ambiental indica que al generar procesos o productos amigables con el medio ambiente existe la posibilidad de incrementar las exportaciones, para el caso en estudio se ha encontrado que los niveles de internacionalización de los negocios verdes en Bogotá-región son decepcionantes, lo que puede tener dos lecturas: o bien están satisfechos con su mercado doméstico, o, por el contrario, no logran consolidarse lo suficiente en el mercado nacional para, posteriormente, dar el paso a los extranjeros, pero esto solo puede ser determinado por futuras investigaciones.

En cuanto al uso de apoyos o programas de fomento a la exportación, pese a que los resultados no son concluyentes, los altos niveles de falta de conocimientos sobre estos podrían sugerir que se requiere que las instituciones que los desarrollan, como, por ejemplo, Bancoldex y ProColombia, propendan a buscar formas de acercarse al pequeño y mediano empresario, en vez de esperar a ser consultadas o, por lo menos, deben ampliar los canales de divulgación. El hecho de que diversos estudios ratifiquen que el financiamiento no es la principal ayuda que buscan los exportadores debería conllevar a plantear nuevos o diferentes mecanismos de apoyo desde Bancoldex.

Aunque institucionalmente existen diversas iniciativas que tienen por objetivo fomentar el crecimiento y la consolidación de los negocios verdes en Colombia, para luego buscar su internacionalización, es adecuado revisar si existe un trabajo coordinado entre agencias o si son esfuerzos aislados que no logran generar el impacto deseado, tal como lo demuestran los resultados del presente documento.

$\mathrm{Si}$ bien los apoyos propuestos por las iniciativas gubernamentales son importantes para lograr que los negocios verdes se formalicen y consoliden, es necesario que los instrumentos se orienten más hacia la promoción de las empresas en el mercado exterior. El objetivo de hacer del crecimiento verde una senda de desarrollo para el país es plausible, sin embargo, se evidencia la necesidad de acercarse más al empresario y brindarle ayuda dirigida al conocimiento de los mercados internacionales y, más aún, brindar asesoría para que logre vencer los temores que lo mantienen en estado de inercia respecto al mercado.

Las perspectivas de investigación son amplias. La metodología usada implica que queden varios asuntos sin respuesta al profundizar secuencialmente en la descripción y el análisis de la información recolectada. En el caso particular de este estudio, asuntos como, por ejemplo, las razones del bajo desempeño exportador, ameritan una medición más exhaustiva para encontrar los determinantes de este comportamiento. Por otra parte, este trabajo se puede replicar a nivel nacional con el ánimo de realizar un diagnóstico mucho más completo sobre el sector a nivel nacional, además de que se puede replantear el instrumento de recolección de información de forma tal que, por ejemplo, sea posible establecer si hubo incremento en el valor, en el volumen y en el destino de las exportaciones a partir del uso de apoyos. 


\section{Referencias}

Álvarez, R. (2004). Sources of export success in small and medium-sized enterprises: the impact of public programs. International Business Review, 13(3), 383-400. DOI: https:// doi.org/10.1016/j.ibusrev.2004.01.002

Analdex. (2018). Informe de exportadores colombianos en el año 2017. Recuperado de https://bit.ly/3nVySmm

Antonietti, R.; Marzucchi, A. (2014). Green tangible investment strategies and export performance: a firm-level investigation. Ecological Economics, (108), 150-161. DOI: https://doi. org/10.1016/j.ecolecon.2014.10.017

Aragón-Correa, J. A.; Hurtado-Torres, N.; Sharma, S.; García-Morales, V. J. (2008). Environmental strategy and performance in small firms: a resource-based perspective. Journal of environmental management, 86(1), 88-103. DOI: https://doi.org/10.1016/j.jenvman.2006.11.022

Ayob, A.H.; Freixaned, J. (2014). Insights into public export promotion programs in an emerging economy: the case of Malaysian SMEs. Evaluation and Program Planning, 46, 36-46. DOI: https://doi.org/10.1016/j.evalprogplan.2014.05.005

Broocks A.; Van Biesebroeck, J. (2017). The impact of export promotion on export market entry. Journal of International Economics, 107, 19-33. DOI: https://doi.org/10.1016/j. jinteco.2017.03.009

Calle, A. M.; Tamayo, V. M. (2005). Estrategia e internacionalización en las pymes: caso Antioquia. Cuadernos de Administración, 18(30), 137-164.

Cansino, J. M., López-Melendo, J.; Pablo-Romero, M. P.; SánchezBraza, A. (2013). An economic evaluation of public programs for internationalization: the case of the diagnostic program in Spain. Evaluation and Program Planning, 41, 38-46. DOI: https://doi.org/10.1016/j.evalprogplan.2013.07.002

Chen, Y. S.; Chang, C. H. (2012). Enhance green purchase intentions: the roles of green perceived valued, green perceived risk, and green trust. Management Decision, 50(3), 502-520. DOI: https://doi.org/10.1108/00251741211216250

Chen, Y. S.; Chang, C. H. (2013). The determinants of green product development performance. Journal of Business Ethics, 116(1), 107-119. DOI: https://doi.org/10.1007/s10551012-1452-x

Comisión Económica para América Latina y el Caribe. (2017). Ecoinnovación y producción verde: una revisión sobre las políticas de América Latina y el Caribe. Santiago de Chile: Cepal.

deVries,H.J.; Bayramoglu,D.K.; van derWiele, T.(2012). Business and environmental impact of ISO 14001. The International Journal of Quality \& Reliability Management, 29(4), 425-435. DOI: https://doi.org/10.1108/02656711211224866
Departamento Nacional de Planeación. (2018). Documento CONPES 3934 - Politica de crecimiento verde. Recuperado de https://bit.ly/3ttUB5Z

Durmuşoğlu, S. S.; Apfelthaler, G.; Zamantili, D.; Alvarez, R.; Mughan, T. (2012). The effect of government-designed export promotion service use on small and medium-sized enterprise goal achievement: a multidimensional view of export performance. Industrial Marketing Management, 41(4), 680-691. DOI: https://doi.org/10.1016/j. indmarman.2011.09.016

Ferreira-Dornela, B.; Teixeira-Carneiro, J. M. (2018). A multiperspective examination of export promotion programs: the case of Peiex by Apex-Brasil. Revista Ibero-Americana de Estratégia, 17(2), 50-63. DOI: https://doi.org/10.5585/riae. v17i2.2562

Freixanet, J. (2012). Export promotion programs: their impact on companies' internationalization performance and competitiveness. International Business Review, 21(6), 10651086. DOI: https://doi.org/10.1016/j.ibusrev.2011.12.003

Garces, M.; Hourneaux-Junior, F.; Farah, D. (2016). Green plastics: analysis of a firm's sustainability orientation for innovation. Revista de Gestão Ambiental e Sustentabilidade, 5(3), 21-36. DOI: https://doi.org/10.5585/geas.v5i3.661

González-Benito, J.; González-Benito, O. (2006). A review of determinant factors of environmental proactivity. Business Strategy and the Environment, 15(2), 87-102. DOI: https:// doi.org/10.1002/bse.450

Kanda, W.; Hjelm, O.; Mejía-Dugand, S. (2016). Promoting the export of environmental technologies: an analysis of governmental initiatives from eight countries. Environmental Development, 17, 73-87. DOI: https://doi.org/10.1016/j. envdev.2015.09.009

Kanda, W.; Mejía-Dugand, S.; Hjelm, O. (2015). Governmental export promotion initiatives: awareness, participation, and perceived effectiveness among Swedish environmental technology firms. Journal of cleaner production, 98, 222-228. DOI: https://doi.org/10.1016/j.jclepro.2013.11.013

Kashmanian, R.; Keenan, C.; Wells, R. (2010). Corporate environmental leadership: drivers, characteristics, and examples. Environmental Quality Management, 19(4), 1-20. DOI: https://doi.org/10.1002/tqem.20259

Langseth, H.; O'Dwyer, M.; Arpa, C. (2016). Forces influencing the speed of internationalization. Journal of Small Business and Enterprise Development, 23(1), 122-148. DOI: https://doi. org/10.1108/JSBED-10-2013-0155 
Lannelongue, G.; González-Benito, O.; González-Benito, J. (2014). Environmental motivatión: the pathway to complete environmental management. Journal of Business Ethics, 124(1), 135-147. DOI: https://doi.org/10.1007/s10551-013-1854-4

Leonidou, L.C.; Fotiadis, T.A.; Christodoulides, P.; Spyropoulou, S.; Katsikeas, C. S. (2015). Environmentally friendly export business strategy: its determinants and effects on competitive advantage and performance. International Business Review, 24(5), 798-811. DOI: https://doi.org/10.1016/j. ibusrev.2015.02.001

Luostarinen, R. (1979). Internationalization of a firm: an empirical study of the internationalization of firm with small and open domestic markets with special emphasis on lateral rigidity as a behavioral characteristic in strategic decision-making. Helsinki: The Helsinki School of Economics.

Martín-Tapia, I.; Aragón-Correa, J. A.; Senise-Barrio, M. E. (2008). Being green and export intensity of SMEs: The moderating influence of perceived uncertainty. Ecological Economics, 68(1-2), 56-67. DOI: https://doi.org/10.1016/j. ecolecon.2008.01.032

Marulanda-Valencia, F.; Morales-Gualdrón, S. (2016). Entorno y motivaciones para emprender. Revista Escuela de Administración de Negocios, (81), 12-28. DOI: https://doi. org/10.21158/01208160.n81.2016.1556

Ministerio de Ambiente y Desarrollo Sostenible. (2014a). Plan Nacional de Negocios Verdes. Bogotá: Ministerio de Ambiente y Desarrollo Sostenible.

Ministerio de Ambiente y Desarrollo Sostenible. (2014b). Programa Regional de Negocios Verdes. Bogotá: Asocars.

Nguyen, T.V.; Le, N.T.; Bryant, S. E. (2013). Sub-national institutions, firm strategies, and firm performance: a multilevel study of private manufacturing firms in Vietnam. Journal of World Business, 48(1), 68-76. DOI: https://doi. org/10.1016/j.jwb.2012.06.008

Porter, M.; van der Linde, C. (1995). Toward a new conception of the environment-competitiveness relationship. The Journal of the Economic Perspectives, 9(4), 97-118. DOI: https://doi. org/10.1257/jep.9.4.97

Programa de las Naciones Unidas para el Desarrollo. (2016). Objetivo 12. Producción y consumo responsables. Recuperado de https://bit.ly/3haSTnQ

Rodríguez, L.; Ríos-Osorio, L. (2016). Evaluación de sostenibilidad con metodología GRI. Dimensión Empresarial, 14(2), 73-89. DOI: https://doi.org/10.15665/rde.v14i2.659

Scheyvens, R.; Banks , G.; Hughes, E. (2016). The private sector and the SDGS: the need to move beyond «business as usual». Sustainable Development, 24(6), 371-382. DOI: https://doi. org $/ 10.1002 /$ sd. 1623

Sierra, J. H. (2009). Assessing exporting culture in Colombian SMES: a look at the export promotion program (EPP). Cuadernos de Administración, 22(39), 99-134.
Spence, M.; Boubaker, J.; Ondoua, V, (2011). Sustainable entrepreneurship is entrepreneurial will enough? A NorthSouth comparison. Journal of Business Ethics, 99(3), 335-367. DOI: https://doi.org/10.1007/s10551-010-0656-1

Tan, A.; Brewerb. P.; Lieschb P. (2018). Rigidity in SME export commencement decisions. International Business Review, 27(1), 46-55. DOI: https://doi.org/10.1016/j. ibusrev.2017.05.002

United Nations Development Group. (2014). Delivering the post2015 development agenda: opportunities at the national and local levels. Recuperado de https://bit.ly/3vSVTZM

Vélez-Ocampo, J.; González-Pérez, M. A. (2015). International expansion of Colombian firms: understanding their emergence in foreign markets. Cuadernos de Administración, 28(51), 189-215. DOI: https://doi.org/10.11144/Javeriana. cao28-51.iecf

Volpe, C.; Carballo, J. (2010). Beyond the average effects: the distributional impacts of export promotion programs in developing countries. Journal of Development Economics, 92(2), 201-214. DOI: https://doi.org/10.1016/j. jdeveco.2009.02.007

Volpe, C.; Carballo, J.; García, P. M. (2012). Public programs to promote firms' exports in developing countries: are there heterogeneous effects by size categories? Applied Economics, 44(4), 471-491. DOI: https://doi.org/10.1080/00036846.2 010.508731

Wilkinson, T.; Brouthers, L. E. (2006). Trade promotion and SME export performance. International Business Review, 15(3), 233252. DOI: https://doi.org/10.1016/j.ibusrev.2006.03.001 